\title{
Lightweight Design of Door Based On Multi-Objective and Robustness
}

\author{
Zhen WANG*, Jie GAO, Hui-Xia LIU and Xiao WANG
}

\author{
School of Mechanical Engineering, Jiangsu University, 212013 Zhenjiang, China \\ *18252585011@163.com
}

\begin{abstract}
Keywords: Vehicle door, Lightweight, Approximate model, Multi-objective optimization,
\end{abstract} Robustness.

\begin{abstract}
Aiming at the problem that the study of vehicle door lightweight is mostly the singleobjective optimization, and do not take unconcern factors into consideration, the vehicle door lightweight research of multi-objective optimization and robustness analysis is conducted. Firstly, the sensitivity analysis is used to pick out vehicle door parts which have more contribution toward vehicle door performance as the design variables, then establishing the approximate models of each vehicle door response. Secondly, taking vehicle door torsion stiffness, drooping stiffness, lateral bending stiffness and dent resistance as the constrains, taking the minimum mass and maximum first three modal frequency of vehicle door as targets, the multi-objective optimization is conducted by using the NSGA- II, then the quality level and reliability analysis is carried out by using Monte Carlo simulation. Thirdly, the vehicle door not only realize the lightweight, reducing $0.97 \mathrm{~kg}$, but also has the good robustness.
\end{abstract}

\section{Introduction}

With the car demand increasing yearly, automobile industry promotes economic development and at the same time it also brings the problem of environmental pollution and the depletion of nonrenewable resources. However, research on weight lightening is an effective method to achieve environmental protection and energy saving. Door which have a larger proportion in body weight is an important body in the opening and closing parts. Lightweight study on door will not only reduces the body quality and has some references in research of vehicle weight $[1,2]$.

Lightweight door structure study at this stage mostly is a deterministic optimization problem, often in constraint conditions to find the optimal solution [3,4,5]. Hao in Hunan University use sensitivity analysis method for single door light, under the premise of ensuring the stiffness and the first order modal increase to realize door lighter 3.4\% [6]. With the goals of first step mode, stiffness and quality, Peng in Wuhan Institute of Technology make multi-objective lightweight design for door, under the premise of ensuring performance requirements to realize door lighter [7]. However, there is little research on the lightweight design of multi-objective and robust door which take into account uncertainties.

This article applied multi-objective optimization and robustness analysis to the door structure of lightweight design. With the constraint of door torsion stiffness, sagging stiffness, lateral bending rigidity and dent resistance meeting the requirements, with the goals of quality minimum and first three modal frequency maximum in door, with the technology of experimental design, response surface approximation model, NSGA-II and Monte Carlo simulation techniques, we have a lightweight design for door structure using multi-objective optimization and robustness analysis.

\section{Optimized Design Process of Door Structure}

Door is based on optimization design and robustness validation method. Under the premise of ensuring door structure of stiffness requirements and modal properties, with the goals of quality minimum and first three modal frequency maximum in door, combined with parameter experiment method, optimal Latin Super cubic design method, response surface method, NSGA-II genetic algorithm, $6 \sigma$ robustness design method and Monte Carlo simulation, we have a lightweight design 
for door structure using multi-objective optimization and robustness analysis. Optimized design processes of door structure are as follow:

(1) Establish finite element model of door structure by Hypermesh.

(2) Based on the sensitivity analysis Pareto chart and choose the final design variables

(3) Use optimal design method of Latin hypercube to gain samples.

(4) Establish the response surface approximation model and shorten the calculation cycle.

(5) Use NSGA-II genetic algorithms to make multi-objective optimization for door structure.

(6) Use Monte Carlo simulation method to analysis quality level and reliability of door.

Lightweight door with good robustness of the structure optimization processes are as shown in Figure 1.

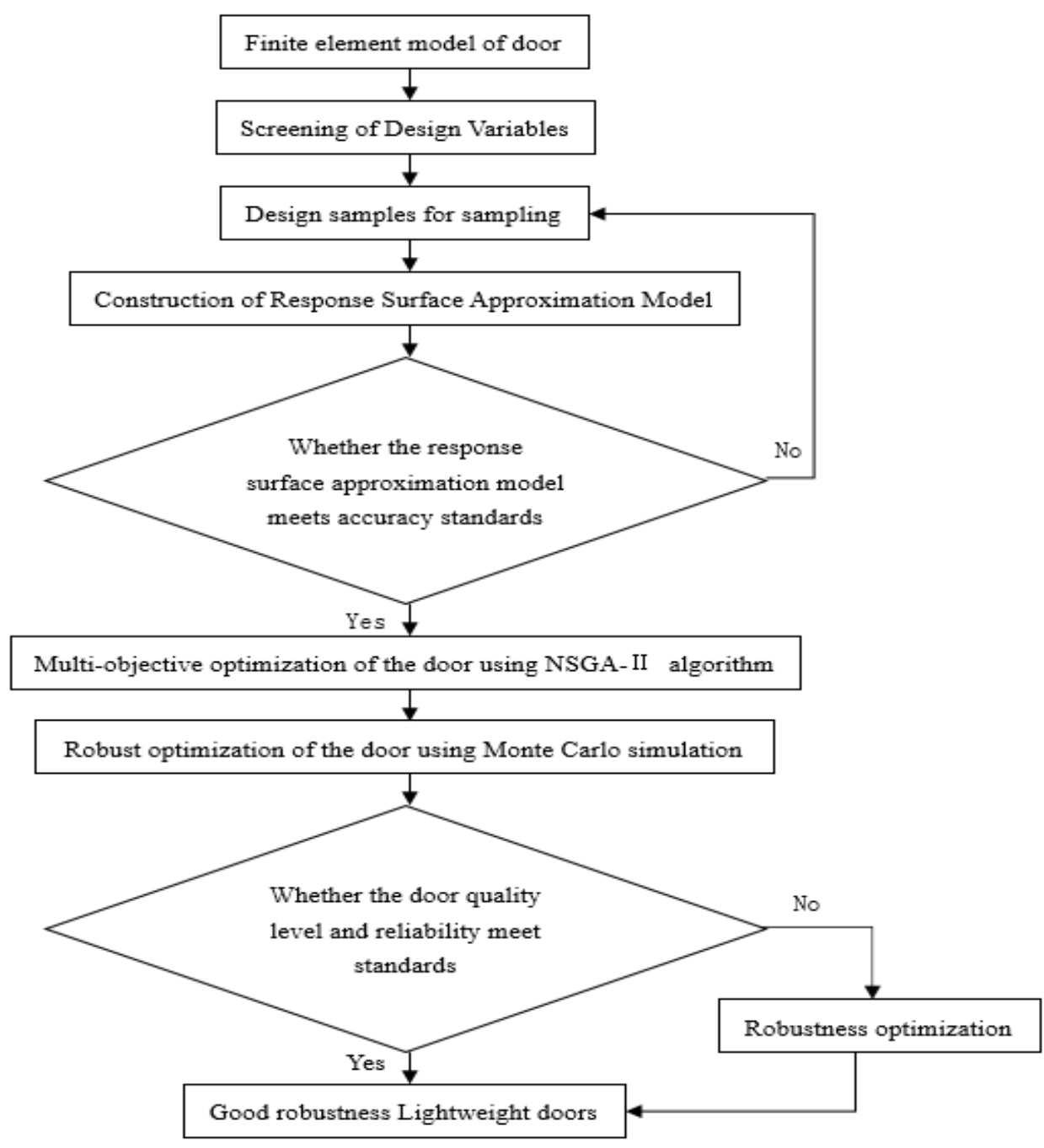

Figure 1. Door structure optimization process

\section{Lightweight Door Based on Multi-Objective Optimization and Robust Analysis}

\section{Finite Element Model of Door Structure}

Finite element model of the door composed of many parts. Each part of the cell size is 10MM around, and is connected by welding, bolts or glue respectively. Three connection modes are simulated in HyperMesh using Cweld, Seam or Adhesives unit respectively.

Torsion stiffness of the door is divided into upper and lower torsional stiffness. Upper and lower torsional stiffness are in the same constraints. In the upper and lower hinge position it offers constraints of six degrees of freedom while in the door lock position it offers constraints of three translational degrees of freedom. The loading condition of upper torsional stiffness is to offer $900 \mathrm{~N}$ 
of $y$-axis at the position of the rear of door frame. However, the lower one is at the position of inner plate fillet [8]. Upper torsional stiffness on the finite element model is shown in Figure 2.

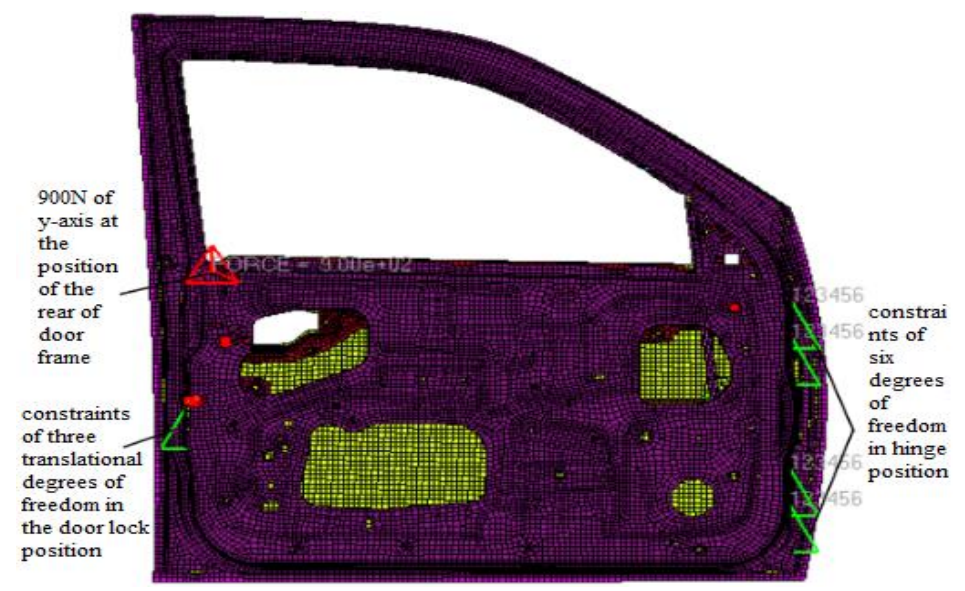

Figure 2. Finite element model of upper torsional stiffness

Door sagging stiffness is divided into $38^{\circ}$ and $58^{\circ}$. The two stiffness have the same constraints and loading conditions. One of the constraints is to constrain the six degrees of freedom in all truncated positions of the quarter-car model, while the loading condition is a Z-direction force of 300 $\mathrm{N}$ applied to the door lock position [1]. $38^{\circ}$ sagging stiffness of finite element model is shown in Figure 3.

The constraint method of door anti-concave analysis is the same as that of torsion stiffness. The loading conditions are a force of $40 \mathrm{~N}$ perpendicular to the indenter at the center of the indenter [9]. The finite element model of door anti-concave analysis is shown in Figure 4.

The lateral bending stiffness of the door is in the same way as the torsional rigidity of the door, and the loading condition is that the Y-direction force of $200 \mathrm{~N}$ is applied to the rear of the door and the lateral bending stiffness of the door is shown in Figure 5.

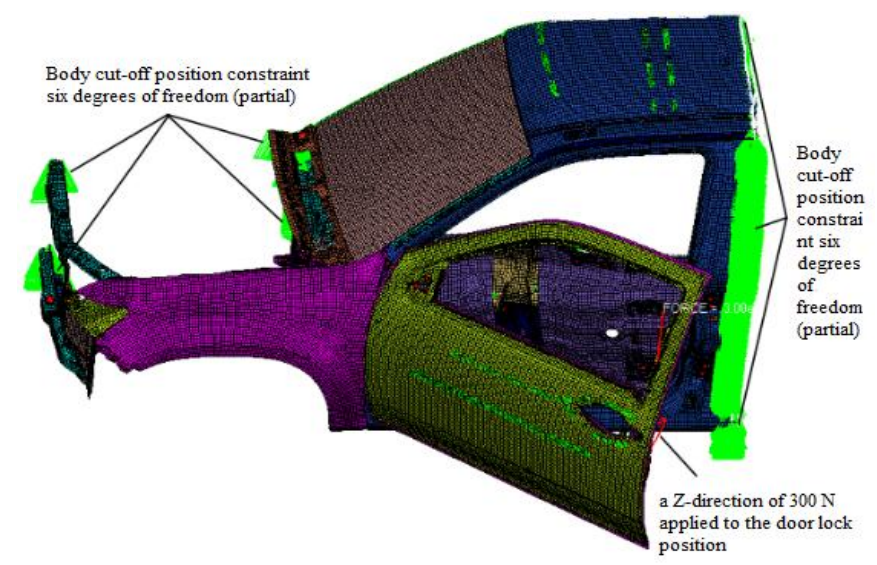

Figure 3. Finite element model of $38^{\circ}$ sagging stiffness 


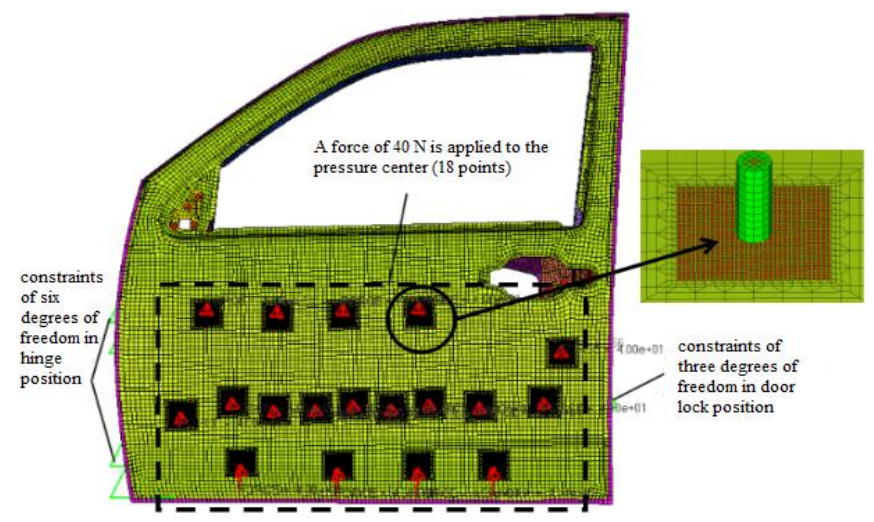

Figure 4. Finite element model of door anti-concave analysis

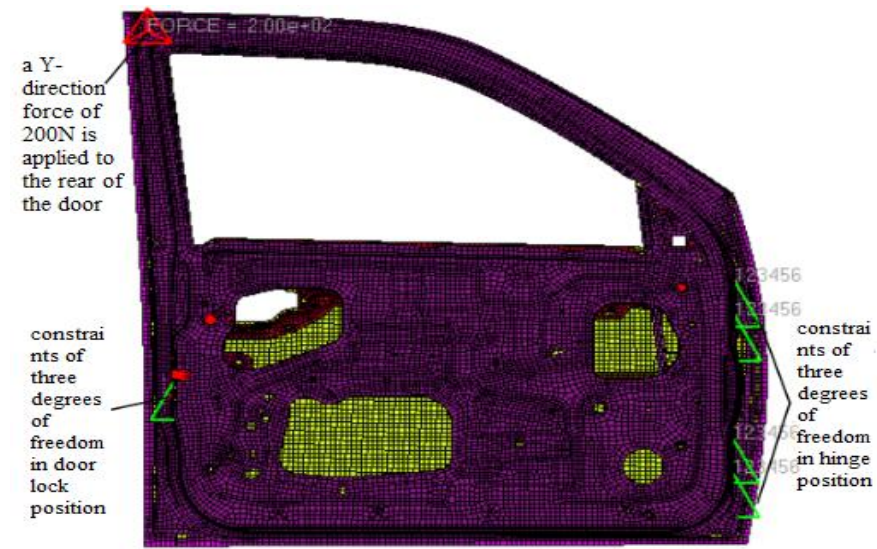

Figure 5. Finite element model of lateral bending rigidity of door

Since the modal analysis of the door is a free mode, no constraint is required.

\section{Screening of Design Variables}

The whole door structures of the parts are so much. This paper selects the door performance of the greater impact on the thickness of the part as a design variable. Figure 6 for the initial selection of 13 design variables.

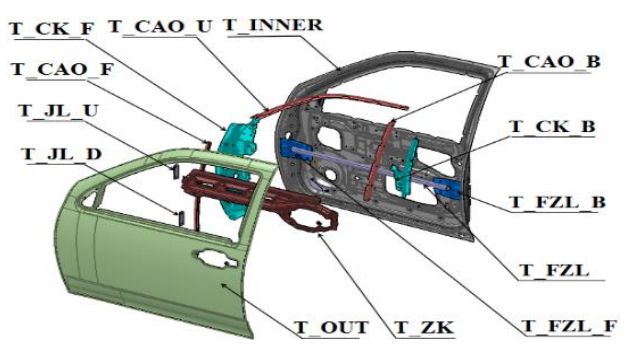

Figure 6. The initial design variables

The initial design variables are related to door torsional rigidity, door sag stiffness, door side bending stiffness, door front third-order mode frequency and door anti-concave performance. But the effect of each design variable is inconsistent, we need to use parametric design method to filter out the design variables that have a great influence. Generally, two levels are taken for each design variable, and other variables are kept at the initial values. As the initial selection of the design variables is 13, so we need 27 samples. The contribution of each main effect is shown in Figure 7 , which is the Pareto graph of each design variable for different response functions. The main variables are shown below: the lower torsional rigidity $\left(\mathrm{y}_{1}\right)$, the upper torsional stiffness $\left(\mathrm{y}_{2}\right)$, the $38^{\circ}$ sagging stiffness ( $\left.\mathrm{y}_{3}\right)$, the $58^{\circ}$ sagging stiffness ( $\left.\mathrm{y}_{4}\right)$, lateral bending rigidity ( $\left.\mathrm{y}_{5}\right)$ and anti-concave analysis point $\left(\mathrm{y}_{6}\right)$, the door mass minimum $(\mathrm{M})$ and the first three order modal frequencies $\left(\mathrm{f}_{1}, \mathrm{f}_{2}, \mathrm{f}_{3}\right)$ 


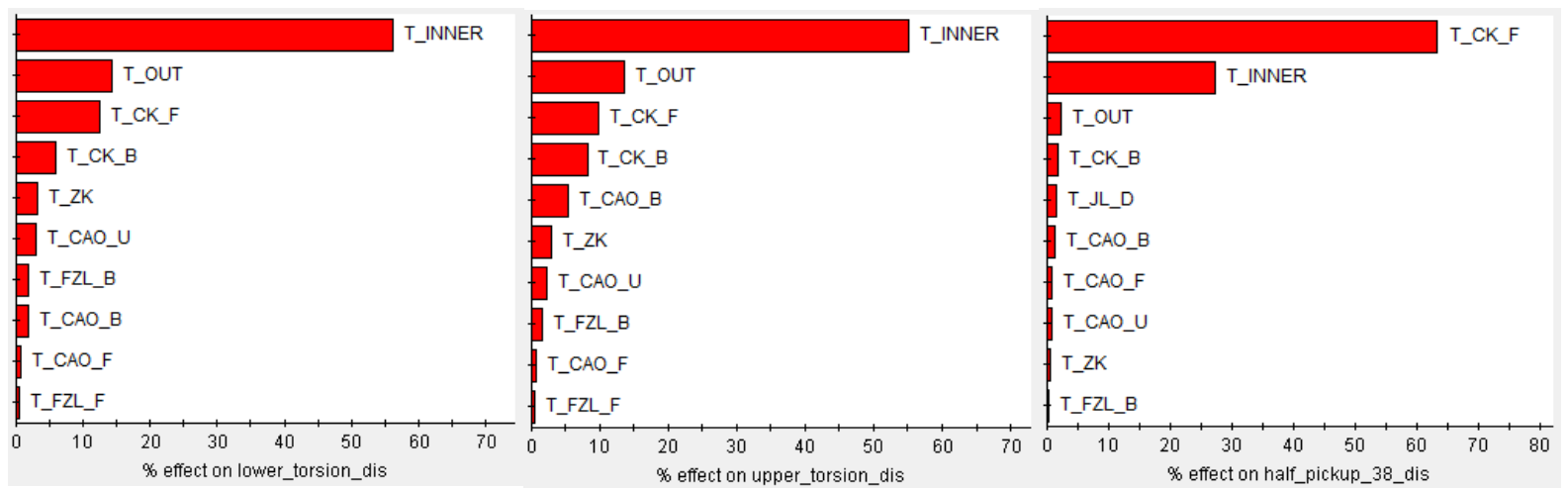

(a) Lower torsional stiffness

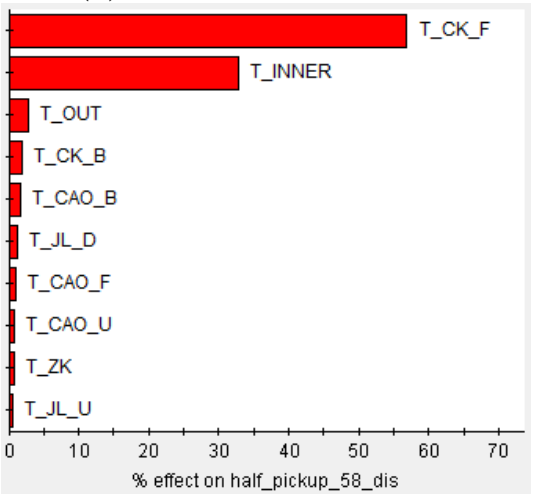

(b) Upper torsional stiffness

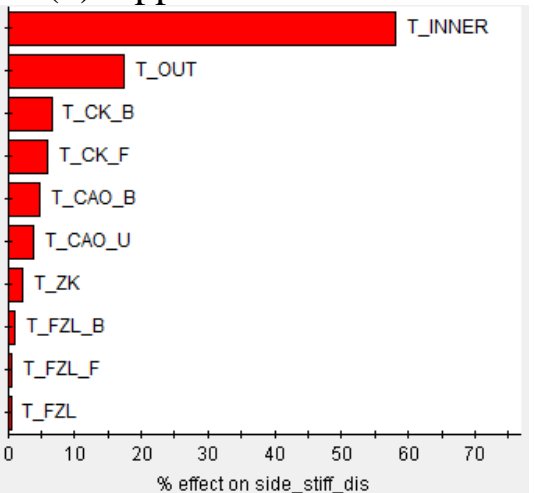

(e) Lateral bending rigidity (c) $38^{\circ}$ Sagging stiffness

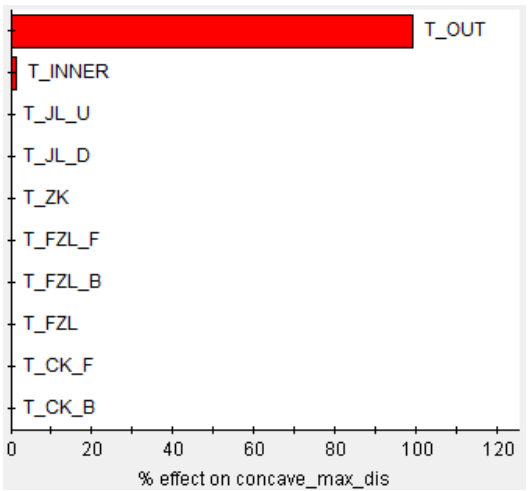

(f) Anti-concave

(d) $58^{\circ}$ Sagging stiffness

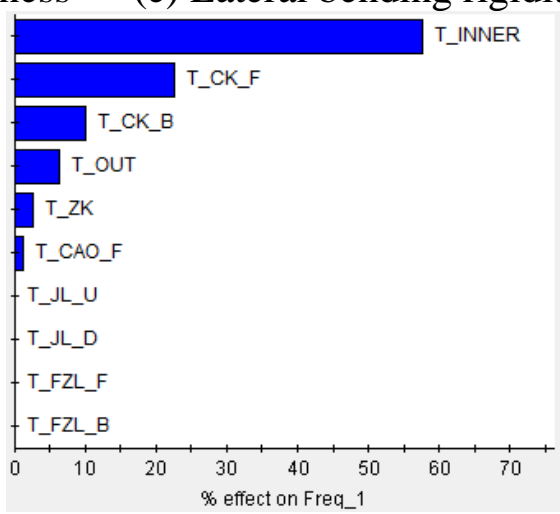

(g) First order mode frequency

Figure 7. The Pareto Graphs of Design Variables to Different Response Functions

From Figure 7, the influence of T_CAO_B, T_CAO_F, T_FZL, T_FZL_B and T_FZL_F is small compared with the other eight design variables. These five design variables can be eliminated and the remaining parts can be used as the design variables after filtering.

\section{Construction of Response Surface Approximation Model}

In order to improve the accuracy of the approximation model, the sampling frequency is $(n+1)(n$ $+2) / 2+2 \mathrm{n}$, where $\mathrm{n}$ is the number of design variables, at least 61 times sampling test. In order to improve the accuracy of the approximate model, 70 sample points are collected in this paper. Finally, an approximate model of design variables and door quality, door torsional rigidity, door sag stiffness, door lateral bending rigidity, door anti-concavity and door modal frequency are constructed [10]. The mathematical expression of the design variable and door quality is:

$$
\mathrm{M}=0.0060+0.0011 \mathrm{X}_{3}+0.0079 \mathrm{X}_{4}+0.0074 \mathrm{X}_{7}+0.0012 \mathrm{X}_{8}
$$

In Equation (1), $X_{3}$ represents T_CK_F, $X_{4}$ represents T_INNER, $X_{7}$ represents T_OUT and $X_{8}$ 
represents T_ZK.

After establishing the response surface approximation model in Isight, it is judged whether the accuracy is up to the standard by determining the coefficient $\left(\mathrm{R}_{\mathrm{G}}{ }^{2}\right)$, where $\mathrm{R}_{\mathrm{G}}{ }^{2}$ is closer to 1 and the numerical value is 0.9 . The fitting accuracy parameters of each response of the door are as shown as below: $0.985 \mathrm{M}, 0.998 \mathrm{y}_{1}, 0.997 \mathrm{y} 2,0.983 \mathrm{y}_{3}, 0.992 \mathrm{y}_{4}, 0.1 \mathrm{y}_{5}, 0.989 \mathrm{y}_{6}, 0.999 \mathrm{f}_{1}, 0.999 \mathrm{f}_{2}, 0.1 \mathrm{f}_{3}$. And the accuracy of the model can be up to standard after the observation.

\section{Multi-Objective Optimization Design Of Door}

In this paper, the thickness of eight door parts is selected as the design variable, and $\mathrm{y}_{1}, \mathrm{y}_{2}, \mathrm{y}_{3}, \mathrm{y}_{4}$, $\mathrm{y}_{5}$ and $\mathrm{y}_{6}$ are taken as the constraint conditions, and the goal is $\mathrm{M}$ and to maximize $\mathrm{f}_{1}, \mathrm{f}_{2}, \mathrm{f}_{3}$. The optimization mathematical model is as follows:

$\operatorname{Obj} . \operatorname{Min}(\mathrm{M}) ; \operatorname{Max}\left(\mathrm{f}_{1}\right) ; \operatorname{Max}\left(\mathrm{f}_{2}\right) ; \operatorname{Max}\left(\mathrm{f}_{3}\right)$

S.t. $\mathrm{y}_{1} \leq 6 \mathrm{~mm}, \mathrm{y}_{2} \leq 6 \mathrm{~mm}, \mathrm{y}_{3} \leq 5 \mathrm{~mm}, \mathrm{y}_{4} \leq 5 \mathrm{~mm}, \mathrm{y}_{5} \leq 6 \mathrm{~mm}, \mathrm{y}_{6} \leq 6 \mathrm{~mm}, \mathrm{f}_{1} \geq 30 \mathrm{~Hz}, \mathrm{f}_{2} \geq 35 \mathrm{~Hz}, \mathrm{f}_{3} \geq 45 \mathrm{~Hz}$

d.v. $\mathrm{X}_{\mathrm{i}}^{(\mathrm{L})} \leq \mathrm{X}_{\mathrm{i}} \leq \mathrm{X}_{\mathrm{i}}^{(\mathrm{U})}, \mathrm{i}=1,2,3 \ldots, 8$

According to NSGA- II algorithm [11], the multi-objective optimization design of door structure is carried out. After 5000 iterations, the solution set of Pareto non-inferior solution is shown in Figure 8.

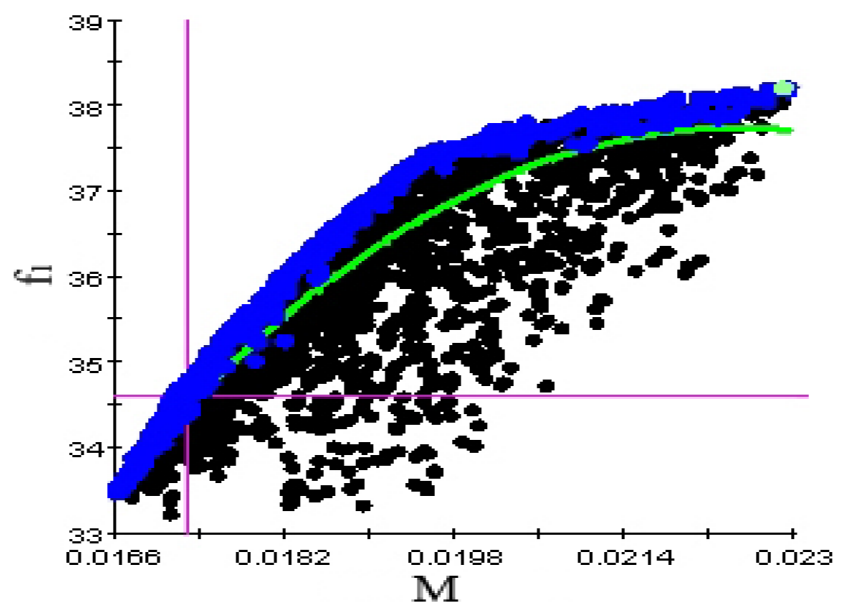

Figure 8. Solution Set of Pareto Non - inferior Solution for Door Mass and First Order Mode Frequency

It can be seen from figure 8 that there is a certain conflict between the two objective functions which the former one is minimization of door quality and the latter one is maximization of the firstorder modal frequency of the door. Therefore, we need to weigh the importance of the two objective functions. According to the optimization experience, from the many non-inferior solutions to select the best solution to meet the optimal design requirements, the data in Table 1 is numerical comparison of door response before and after optimization.

Table 1. Numerical comparison of door response before and after optimization

\begin{tabular}{|l|l|l|c|}
\hline Output response & Initial value & Optimization value & Enterprise standards \\
\hline $\mathrm{M}$ & $18.16 \mathrm{~kg}$ & $17.19 \mathrm{~kg}$ & - \\
\hline $\mathrm{y}_{1}$ & $2.801 \mathrm{~mm}$ & $2.933 \mathrm{~mm}$ & $\leq 6 \mathrm{~mm}$ \\
\hline $\mathrm{y}_{2}$ & $4.392 \mathrm{~mm}$ & $4.522 \mathrm{~mm}$ & $\leq 6 \mathrm{~mm}$ \\
\hline $\mathrm{y}_{3}$ & $4.396 \mathrm{~mm}$ & $3.954 \mathrm{~mm}$ & $\leq 5 \mathrm{~mm}$ \\
\hline $\mathrm{y}_{4}$ & $4.765 \mathrm{~mm}$ & $4.370 \mathrm{~mm}$ & $\leq 5 \mathrm{~mm}$ \\
\hline $\mathrm{y}_{5}$ & $5.006 \mathrm{~mm}$ & $5.401 \mathrm{~mm}$ & $\leq 6 \mathrm{~mm}$ \\
\hline $\mathrm{y}_{6}$ & $1.019 \mathrm{~mm}$ & $1.938 \mathrm{~mm}$ & $\leq 3 \mathrm{~mm}$ \\
\hline $\mathrm{f}_{1}$ & $34.32 \mathrm{~Hz}$ & $34.59 \mathrm{~Hz}$ & $\geq 30 \mathrm{~Hz}$ \\
\hline $\mathrm{f}_{2}$ & $36.84 \mathrm{~Hz}$ & $36.18 \mathrm{~Hz}$ & $\geq 35 \mathrm{~Hz}$ \\
\hline $\mathrm{f}_{3}$ & $52.24 \mathrm{~Hz}$ & $57.57 \mathrm{~Hz}$ & $\geq 45 \mathrm{~Hz}$ \\
\hline
\end{tabular}


As it can be seen from Table 1, the quality of the optimized door is $17.19 \mathrm{~kg}$, which is $0.97 \mathrm{~kg}$ less than the optimized door quality, and the reduction range is $5.34 \%$. The first and third order modal frequencies of the optimized door are $34.59 \mathrm{~Hz}$ and $57.57 \mathrm{~Hz}$, respectively, which are not only $0.27 \mathrm{~Hz}$ and $5.33 \mathrm{~Hz}$ higher than the pre-optimization value, but also meet the requirements of more than $30 \mathrm{~Hz}$ and $45 \mathrm{~Hz}$. The modal frequency of the second order of the door is reduced by $0.74 \mathrm{~Hz}$, and the amplitude of the second-order door is smaller than that before the optimization. Because the first three-stage door mode frequency is hard to reach the same goal, only the first-order mode frequency is better. The idling frequency of the motive and the first order modal frequency of the white body are developed. The optimized door not only satisfies the enterprise standard of stiffness performance and anti-concavity, but also achieves the goal of first-order modal frequency increase and door lightening. However, considering the interference of uncertain factors, it is necessary to further carry out the robustness of the door inspection and analysis.

\section{Analysis of Vehicle Door Quality Level and Reliability Based on Robustness \\ Quality and Reliability Analysis of Sampling Methods}

Monte Carlo simulation is a method of dealing with numerical problems using random numbers. In the case of instability, it is usually used to extract the sample points to study the quality and reliability of the optimal solution set, in which the sampling method of quality and reliability analysis is divided into simple random sampling and descriptive sampling. Because the sample sampling method is less, and the quality of statistical analysis is better, the description sampling method is adopted to analyze the quality and reliability.

\section{Based on the Robustness of Door Quality Level and Reliability Analysis Results}

In this paper, the quality and reliability of the output response are analyzed based on the robustness of the multi-objective optimization design of the door. The thickness of the eight design variables is taken as the uncertainty factor and the variation coefficient is 0.05 . Based on the approximate model of the door structure, Carlo simulation of descriptive sampling method to extract the sample points, the number is 1000 . And the door output response quality levels are all $8 \sigma$, and the reliabilities are all 1 , the multi-objective optimization door has good robustness, and there's no need to carry out $6 \sigma$ robust optimization.

\section{Summary}

This chapter is based on multi-objective optimization and robustness analysis to do some researches on lightweight of the door structure. The main contents of the research are as follows:

(1) Based on the design variables and the Pareto diagram of different response functions, the design variables were selected from the 13 selected design variables to sample the sample points, which laid a foundation for constructing the response surface approximation model.

(2) Based on the approximation model of door output response and NSGA- II multi-objective optimization, a multi-objective optimization mathematical model of the door structure is constructed, and the solutions of the Pareto non-inferior solutions are obtained. And the optimal solution satisfying the optimization condition is selected.

(3) The descriptive sampling technique in Monte Carlo simulation is used to analyze the door quality level and reliability after multi-objective optimization. The quality level and reliability of each output response are $8 \sigma$ and 1 respectively. After optimization of the door has a good robustness. The study can be used as other vehicles in the assembly or parts of the reference method.

\section{References}

1. Haiming Gu, CAE Analysis and Optimum Design of Car Door System (Changchun, HIT, 2013)

2. Fang Lu, Research on the Lightweight Body of White Body Based on Multi-Disciplinary Optimum Design Method (Changchun, JLU, 2014) 
3. Shihua Huang, Aiguo Cheng, Chaohui $\mathrm{Hu}$, Shaowei Chen, Research on lightweight tailored blank door based on $6 \sigma$ robustness J. Auto.Eng. 3, 262-266 (2011)

4. Hongyan Wang, Shaoying Xu, Lightweight door design J. Auto.Eng. 3, 349-353 (2004)

5. Qing Tu, Lijuan Wang, Zongyu Chen, Dongmin Chen, Pengfei Zhao, Lightweight Design of Tailgate Welded Blanking Back Based on Topology Optimization J. Mech.Str. 2,272-276 (2015)

6. Qi Hao, Jiwei Zhang, Study on sensitivity analysis of door structure optimization design J. Auto.Tech. 5, 40-44 (2010)

7. Tao Peng, Analysis and Research on Door System of Minitype Car (Wuhan, WUT, 2013)

8. Xuan He, Torsional Stiffness Analysis and Optimization of the Shape of a GV Door Based on OptiStruct J. Bj.Auto.Eng. 5,39-42 (2014)

9. Yanyun Xing, Tingting Gao, Bo Yu, Rigidity Analysis and Improvement Design of Car Door Sinking J. Tj.Tech.Edu. 2, 25-28 (2009)

10. Khuri A I, Mukhopadhyay S. Response surface methodology J. WIREs. 2, 128-149 (Computational Statistics,2010)

11. Yuan Gao, Research and Application of Non - dominated Sorting Genetic Algorithm (Hangzhou, ZWU, 2006) 